\title{
ANISAKIDÉS ET ANISAKIDOSES HUMAINES
}

\author{
Première partie : Données bibliographiques
}

\author{
Weiyi HUANG*, J. BUSSIÉRAS*
}

\begin{abstract}
RÉSUMÉ. Les auteurs récapitulent les données bibliographiques actuellement disponibles sur les larves d'Anisakidés trouvées chez les poissons marins (genres Anisakis, Pseudoterranova, Hysterothylacium et Contracaecum), ainsi que sur les dangers présentés pour l'homme par la consommation de poissons parasités.

Mots-clés : Anisakidés. Anisakis. Pseudoterranova. Hysterothylacium. Contracaecum. Granulome éosinophilique. Zoonose. Anisakidose.
\end{abstract}

\section{Anisakidae and human anisakidosis: 1. Data from current literature.}

SUMMARY. Currently available data about larvae of Anisakidae (genera Anisakis, Pseudoterranova, Hysterothylacium and Contracaecum) from marine fishes are analysed. Hazard of such parasites for fish-consumers are emphasized.

Key-words : Anisakidae. Anisakis. Pseudoterranova. Hysterothylacium. Contracaecum. Eosinophilic granuloma. Zoonosis. Anisakidosis.

Depuis une vingtaine d'années, une nouvelle affection gastro-intestinale humaine attire de plus en plus l'attention dans le monde. Cette maladie est provoquée par l'ingestion de poissons crus ou peu cuits, qui contiennent des larves vivantes de Nématodes appartenant à la famille des Anisakidés, et que l'on dénomme donc Anisakidose. On emploie fréquemment aussi le terme d' " anisakiase " (en anglais " anisakiasis "), mais nous éviterons cette appellation, qui laisse entendre que les infestations humaines sont toujours dues à des espèces du genre Anisakis, ce qui est inexact, car les parasites responsables appartiennent à trois genres différents au moins.

* Service de Parasitologie, École Nationale Vétérinaire, F 94704 Maisons-Alfort Cedex.

L'essentiel de ce travail est extrait d'un mémoire de D. E. A. de Parasitologie : écologie, pathologie, présenté à l'Université des sciences et techniques du Languedoc, le 28 septembro 1987.

Accepté le 2 février 1988. 
Très répandue au Japon et aux Pays-Bas, cette maladie existe aussi en France. Calvet (1968) a signalé pour la première fois un phlegmon iléal d'origine parasitaire dû à une larve d'Anisakis retrouvée à l'examen anatomo-pathologique. Par la suite, des dizaines de cas ont été rapportés (cités par Mudry et coll., 1986).

A plusieurs reprises, nous avons reçu des larves prélevées dans la chair de poissons commerciaux, ou même dans l'assiette du consommateur, ce qui pose des problèmes sanitaires. Mais l'épidémiologie et les risques liés à ces parasites restent encore mal connus en France, et les publications à ce sujet sont peu nombreuses.

Aussi nous a-t-il paru utile de présenter dans une première partie une revue bibliographique sur cette question. Dans une deuxième partie, nous apporterons les résultats d'une enquête personnelle sur les larves d'Anisakidés en France. Les références bibliographiques seront présentées à la fin de la deuxième partie.

\section{I - Généralités}

\section{1 - DÉFINITION ET HISTORIQUE}

Les Nématodes Anisakidés sont des parasites, à l'état adulte, du tube digestif d'animaux marins (Poissons, Oiseaux, Mammifères). Les larves de troisième stade (L3), ou larves infestantes, sont hébergées par des poissons téléostéens ou des mollusques céphalopodes. Aucune des espèces d'Anisakidés parasites des poissons n'est transmissible à l'homme, en ce sens que ces larves ne peuvent évoluer et devenir adultes chez lui ; néanmoins, leur ingestion, vivantes, peut provoquer des accidents graves (Dollfus, 1970).

Les premiers cas d'anisakidose furent signalés aux Pays-Bas en 1955 par Van Thiel et coll. (1960). Les larves furent d'abord identifiées comme appartenant à l'espèce Eustoma rotundatum, puis rattachées au genre Anisakis par Van Thiel (1962).

De nombreux cas furent ensuite retrouvés :

— au Japon (Oshima, 1972 ; Fujino et coll., 1984, etc.),

- en Corée (Kim et coll., 1971),

- aux États-Unis (Pinkus et coll., 1975; Litte et Macphail, 1972 ; Kliks, 1983, etc.),

- en France (Doby et coll., 1975 ; Cocheton et coll., 1984 ; Godeau et coll., 1985 ; Mudry et coll., 1986, etc.),

- au Danemark (Andreassen, 1970),

- en Angleterre (Lucas et coll., 1985),

- en Nouvelle-Zélande (Paltridge et coll., 1984), etc.

Ces infestations sont encore appelées " maladie du ver des harengs " (due aux larves d'Anisakis simplex), et "maladie du ver de la morue " (due aux larves de Pseudoterranova decipiens), car ces deux espèces de poissons sont souvent très parasitées (Euzéby, 1984). 


\section{2 - SYSTÉMATIQUE}

La systématique des larves d'Anisakidés les plus fréquemment rencontrées chez les poissons destinés à la consommation humaine, est la suivante (Hartwich, 1974) :

Classe : Nematoda.

Ordre : Ascaridida.

Super-famille : Ascaridoidea.

Famille : Anisakidae Skrjabin et Karokhin, 1945.

Sous-famille : Anisakinae Railliet et Henry, 1912.

Genres :

Anisakis Dujardin, 1845 (espèce principale A. simplex Dujardin, 1845).

Pseudoterranova Mosgovoi, 1951 (espèce principale $P$. decipiens Krabbe, 1878). Contracaecum Railliet et Henry, 1912.

Sous-famille : Raphidascaridinae.

Genre :

Hysterothylacium Ward et Magath, 1916.

Mais cette systématique, et la dénomination même des divers genres et espèces, a fait l'objet de nombreuses controverses :

a) Pocr le genre Anisakis, les principaux synonymes ont été récapitulés par Hartwich (1974) :

Anisakis (Skrjabinisakis) Mozogovoi, 1951 ;

Capsularia Zeder, 1800, nec Mooder, 1793 ;

Conocephalus Diesing, 1861, nec Thunberg, 1812 ;

Filocapsularia Deslongchamps, 1842, nom. oblit.;

Peritrachelius Diesing, 1861 ;

Stomachus Goeze in Zeder, 1800, nom. nud.

b) Dans LE Genre Pseudoterranova, la taxinomie compliquée et l'histoire de la nomenclature de l'espèce Pseudoterranova (=Phocanema =Porrocaecum $=$ Terranova) decipiens, ont été étudiées par Gibson (1983); le parasite trouvé chez la morue à l'état larvaire, ou chez les phoques à l'état adulte, a changé plusieurs fois de nom depuis 50 ans. Il fut d'abord appelé Ascaris decipiens, puis il devint Porrocaecum decipiens, puis Terranova decipiens. Myers crée un nouveau genre Phocanema pour ce ver, qui est alors nommé Phocanema decipiens.

Enfin, Gibson (1983) conclut que Phocanema Myers, 1959 est synonyme de Pseudoterranova Mosgovoi, 1951. Cette notion est maintenant bien acceptée, et l'expression Pseudoterranova decipiens (Krabbe, 1878) est couramment utilisée dans la littérature.

c) LE GENRE Hysterothylacium Ward et Magath, 1917 a pour synonymes (Deardorff et Overstreet, 1980) :

Thynnascaris Dollfus 1933, espèce-type T. legendrei Dollfus, 1933, synonyme de Ascaris cornuta; 
Thynnascaris Dollfus 1935, sous-genre de Contracaecum;

Simplexonema Kreis, 1952, sous-genre de Contracaecum, avec C. (S.) cyclopteri Kreis, 1952 comme espèce-type ;

Erschovicaecum Mozgovoi in Skrjabin et coll., 1951 ; sous-genre de Contracaecum, avec $C$. (E.) aduncum (Rudolphi, 1802) comme espèce-type;

Acollaris Araujo, 1970 ; sous-genre de Contracaecum avec C. (A.) marinum (Linné, 1767), comme espèce-type.

d) Les larves DU Genre Contracaecum sont souvent confondues avec celles d'Hysterothylacium, car divers auteurs utilisent encore Contracaecum dans un sens large. Au sens strict, la larve de Contracaecum peut être distinguée par la situation du pore excréteur près de l'interlabium ventral, et par le parasitisme de l'adulte chez les Oiseaux et les Mammifères. Les espèces d'Hysterothylacium sont parasites au stade adulte chez les poissons.

\section{3 - RÉPARTITION GÉOGRAPHIQUE}

La famille des Anisakidés a une distribution très large ; elle se rencontre dans presque tous les océans et toutes les mers, bien qu'en certaines zones puissent manquer quelques espèces (Smith et Wootten, 1978).

Anisakis en particulier est un genre très répandu, notamment dans les eaux froides et les régions polaires; à l'état adulte, il parasite 26 espèces de Cétacés et 12 espèces de Pinnipèdes (Davey, 1971), et plus de 120 espèces de poissons hébergent les L3 d'Anisakis (Euzéby, 1984). Mais il est aussi présent dans les eaux tropicales : Hawaï (Deardorff et coll., 1982), Nouvelle-Zélande (Hurst, 1984), Australie (Cannon, 1977).

\section{II - Étude des parasites}

\section{1 - MORPHOLOGIE LARVAIRE}

La morphologie des adultes a été décrite par Davey (1971) dans le cas du genre Anisakis, mais nous n'étudierons que les caractères des larves, car seules ces dernières nous intéressent. Leur morphologie est bien connue dans les principales espèces grâce aux travaux de nombreux auteurs (Koyama et coll., 1969 ; Petter, 1969 ; Grabda, 1976 ; Cannon, 1977 ; Deardorff et Overstret, 1980 ; Deardorff et coll., 1982 ; Hurst, 1984 ; Smith, 1983 ; Weerasooriya et coll., 1986). Nous présentons ci-dessous les principaux caractères permettant de les identifier, en nous basant principalement sur les descriptions des espèces-types :

a) Anisakis simplex

1) LaRve 3, OU LaRve infestante

Elle a une longueur de 14 à $30 \mathrm{~mm}$, et un diamètre de $0,5 \mathrm{~mm}$. Blanc jaunâtre à l'état frais, on la reconnaît facilement à la loupe et même à l'œil nu par une tache plus blanche située à environ $2 \mathrm{~mm}$ en arrière de l'extrémité antérieure. 
Elie possède à l'extrémité antérieure une dent de pénétration de forme triangulaire, dont la pointe est dirigée ventralement. Sous la cuticule en arrière de la dent, se trouvent trois renflements prélabiaux ou ébauches labiales (une dorsale et deux subventrales) entourant l'orifice buccal.

A l'extrémité postérieure, s'observe un mucron.

CEsophage en deux parties avec un œsophage musculaire (longueur 1,8 à $2,8 \mathrm{~mm}$ ), suivi d'un œsophage glandulaire, ou ventricule, assez allongé, et dont la limite de séparation avec l'intestin est dirigée obliquement (caractéristique de l'espèce $A$. simplex); absence d'appendice œsophagien et de cæcum intestinal.

L'ébauche génitale est invisible à ce stade.

Les coupes histologiques permettent de mettre en évidence la présence d'une glande excrétrice percée d'un canal, située ventralement le long de l'œsophage musculaire, du ventricule et de l'intestin antérieur. Cette glande est visible sur la larve vivante sous l'aspect d'une bande sombre. Le pore excréteur est situé entre la base des deux ébauches labiales subventrales, sous la forme d'une petite fente transversale.

2) Larve 4

L'étude des L4 d'Anisakis simplex a porté sur des spécimens récoltés chez des humains (Fujino et coll., 1984), ou des animaux d'expérience (Gibson, 1970), ou obtenues in vitro par culture (Grabda, 1976). On constate qu'il n'y a presque pas de différence de longueur entre ce stade et le stade précédent.

On distingue les L4 des L3 par les caractères suivants :

1. Perte de la dent et du mucron.

2. Les sillons transversaux de la cuticule deviennent plus foncés et réguliers, donnant l'aspect d'une segmentation.

3. Les trois lèvres sont bien développées, avec papilles apparentes, chaque lèvre possède sur son bord libre une délicate crête denticulée.

4. La lumière intestinale apparaît nettement, comme un canal en zigzag.

5. Ventricule rétréci, incurvé, et, sur la L4 âgée, vide.

6. Appareil génital visible sur la L4 âgée (utérus observable vers la mi-longueur du corps).

\section{b) Pseudoterranova decipiens}

Les L3 sont plus grandes, de $25 \mathrm{~mm}$ à $40 \mathrm{~mm}$ de longueur et de 0,8 à $1,2 \mathrm{~mm}$ de diamètre ; elles ont une couleur rougeâtre. La cuticule est plus épaisse. Le ventricule, cylindrique, ressemble à celui d'Anisakis simplex, mais il est accompagné d'un cæcum intestinal ; les autres caractères rappellent Anisakis simplex.

\section{c) LE GENRE Hysterothylacium}

Les dimensions sont variables selon les espèces, de $1,5 \mathrm{~mm}$ à $25 \mathrm{~mm}$ de longueur et de 50 à $260 \mu \mathrm{m}$ de diamètre ; coloration blanc jaunâtre. Les L3 sont munies d'une dent larvaire moins pointue que chez Anisakis. Les L4 présentent 3 lèvres 
bien développées, dépourvues de crête denticulée. Queue conique, souvent munie de petites épines chez la L4.

Ventricule presque sphérique, appendice œsophagien et cæcum intestinal présents; appendice cesophagien sacciforme ou cylindrique, avec un septum divisant longitudinalement cette structure en deux cordons accolés.

Le pore excréteur est situé au niveau ou à proximité de l'anneau nerveux. Il n'existe pas de glande excrétrice ventrale.

L'appareil génital est bien développé même chez la L3 ; il est constitué d'un (chez le mâle) ou de deux (chez la femelle) cordons de forme sinueuse, qui occupent plus de la moitié postérieure du corps.

\section{d) LE GEnke Contracaecum}

Les L3 ressemblent aux L3 d'Hysterothylacium. On les distingue par la position du pore excréteur qui se trouve à la base des ébauches labiales, comme chez Anisakis, et par le faible développement de l'ébauche génitale.

\section{2 - CYCLE ÉVOLUTIF D'ANISAKIS SIMPLEX}

Le cycle évolutif des Anisakidés n'est pas encore entièrement connu. Dans les cas pour lesquels il a été assez bien étudié (Anisakis, Contracaecum), il apparaît qu'il se déroule selon le schéma suivant, établi pour A. simplex (fig. 1).

Les œufs sont émis non embryonnés par des mammifères marins porteurs d'Anisakis d'adultes, et évoluent dans le milieu extérieur ; l'éclosion libère une L2,

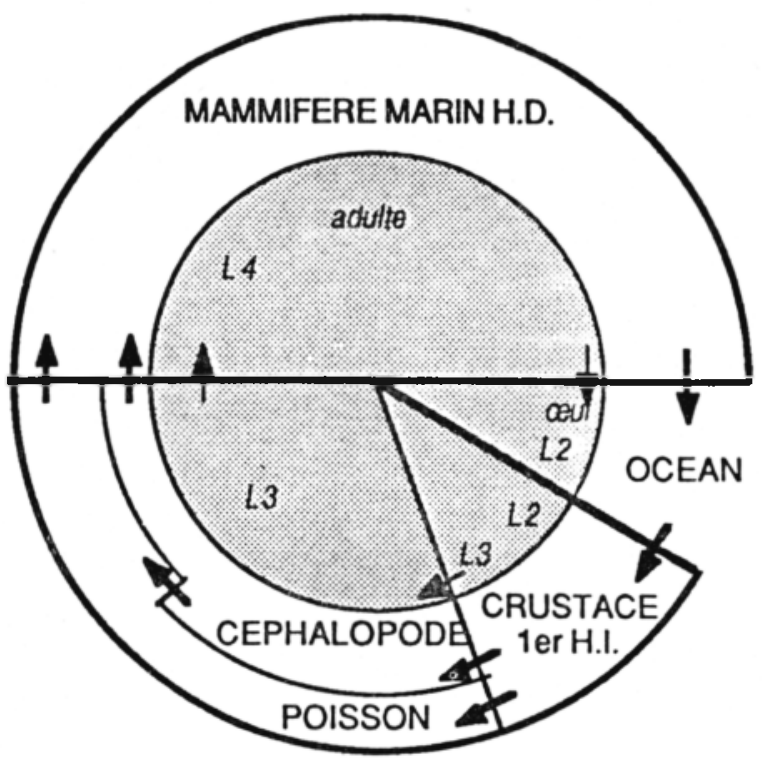

Figure $N^{\circ 1}$ : cycle évolutlf d'Anisakis sp. 
enveloppée dans la dépouille exuviale de la L1. Les L2, ainsi engainées, sont absorbées par des Crustacés Malacostracés hôtes intermédiaires (=H. I.). Chez le crustacé, la L2 se transforme en L3, mais cette dernière demeurerait à un stade subinfestant; si le mammifère hôte définitif ( $=$ H. D.) mange des crustacés parasités, ces L3 ne peuvent pas se développer.

Le stade infestant ne serait atteint que chez un $2^{\mathrm{e}} \mathrm{H}$. I., prédateur de crustacés : poisson ou mollusque céphalopode (calmar). C'est l'ingestion de ces seconds H. I. qui assure l'infestation des H. D. Il est possible que poissons et céphalopodes interviennent, non seulement comme H. I., mais aussi comme hôtes paraténiques : petits poissons ingérés par un céphalopode de grand format, avec paraténie chez le céphalopode, ou petit céphalopode dévoré par un poisson de grand format, avec paraténie chez le poisson. Les L3 infestantes sont ingérées avec leur hôte, par un H. D. (mammifère marin cétacé ou pinnipède). Après deux mues, les vers adultes s'installent dans l'estomac de l'hôte définitif (Smith, 1983; Euzéby,1984).

\section{3 - LOCALISATION DES LARVES D'ANISAKIDÉS CHEZ LES POISSONS}

On observe, en général, les L3 d'Anisakis dans la cavité abdominale, enroulées en spirale, entourées par une capsule; elles sont généralement plaquées à la surface du mésentère ou des gonades, plus rarement du foie ou du tube digestif, chez le hareng et divers autres poissons. Mais on trouve aussi des L3 dans le tissu musculaire. Certains auteurs considèrent que la L3 d'Anisakis n'entre dans le muscle qu'après la mort du poisson (Van 'Thiel et coll., 1960 ; Vilk, 1966). Mais Smith et Wootten (1975) ont montré que chez le hareng frais, il y a déjà $4 \%$ des L3 localisées dans le muscle. De même, Khalil (1969, cité par Smith et Wootten, 1978) observe $2 \%$ de larves musculaires, et Hauck (1977) en trouve 3,5\%.

Après la mort du poisson, si celui-ci est conservé dans des conditions non extrêmes (poissons conservés non congelés, ou seulement salés, fumés, ou marinés), les L3 sortent de leur capsule et pénètrent dans les muscles de la paroi abdominale. Cette migration abdomino-musculaire permet une augmentation du nombre des L3 dans la chair du poisson. Vik (1966) a trouvé que 10 à $13,6 \%$ des L3 ont migré après 3-4 jours. Smith et Wootten (1975) ont montré que $20 \%$ des L3 sont trouvées dans le muscle après 37 heures de conservation à $+10^{\circ} \mathrm{C}$. Hauck (1977) a noté que $50 \%$ des harengs fumés hébergent des L3 d'Anisakis dans leur chair. Panebianco et Schiavo (1985) ont trouvé que 85,7\% des harengs fumés sont parasités par ces mêmes larves.

Chez les merlus, Merluccius spp., les taux d'infestation par les L3 d'Anisakis sont souvent très élevés (cf. II ${ }^{\mathrm{e}}$ partie). Smith (1984) a trouvé que 66,7\% des L3 d'Anisakis sont localisées dans le muscle du merlu au moment de la pêche, $80 \%$ 8 heures plus tard, $100 \% 24$ heures plus tard. Cattan et Carvajal (1984) pensaient que l'éviscération immédiate n'empêchait pas la pénétration des larves dans le muscle, car pour eux les taux d'infestation musculaire sont équivalents à 0 heure, à 15 heures et à 30 heures après la pêche.

Les larves de Pseudoterranova decipiens sont observées souvent dans les muscles. 
Young (1972) a signalé que, parmi les larves trouvées dans le muscle de 5400 morues, $60 \%$ sont des $P$. decipiens, en outre, elles sont réparties dans toute la musculature du poisson, même avant la mort de l'hôte.

Les larves du genre Hysterothylacium (L3 et L4) sont libres dans la cavité abdominale, ou dans le foie, ou dans le tube digestif, mais très rarement trouvées dans les muscles.

\section{4 - RÉSISTANCE DES LARVES}

Les larves d'Anisakidés ont été soumises à l'influence de divers agents extérieurs, après la mort du poisson (Smith et Wootten, 1978). Dans le tableau I, sont récapitulés les divers travaux sur ce sujet.

Tableau I. - Résistance des L3 d'Anisakis.

\begin{tabular}{|c|c|c|}
\hline Condition & Survie des L3 & Auteurs \\
\hline $\begin{array}{l}\text { Froid } \\
4^{\circ} \mathrm{C} \text { à } 10^{\circ} \mathrm{C} \\
0^{\circ} \mathrm{C} \\
-5^{\circ} \mathrm{C} \\
-10^{\circ} \mathrm{C} \text { à }-20^{\circ} \mathrm{C} \\
-20^{\circ} \mathrm{C} \text { chez sébaste }\end{array}$ & $\begin{array}{l}>8 \text { mois } \\
<25 \text { jours } \\
<4 \text { jours } \\
<24 \text { heures } \\
<6 \text { jours }\end{array}$ & $\begin{array}{c}\text { Deardonft } 1984 \\
= \\
= \\
"\end{array}$ \\
\hline $\begin{array}{l}\text { Dans } \mathrm{NaCl} \\
\mathrm{NaCl} \mathrm{sec} \\
\text { Saumure saturée } \\
\text { Solution } 33 \text { p.100 }\end{array}$ & $\begin{array}{l}10 \text { minutes } \\
24 \text { heures } \\
8 \text { jours }\end{array}$ & $\begin{array}{l}\text { Khalil } 1969 \\
\quad \\
\text { Nygard } 1967\end{array}$ \\
\hline $\begin{array}{l}\text { Dans l'acidité } \\
2 \% \text { acide acétique } \\
+5 \% \mathrm{NaCl} \text { à } \mathrm{O}^{\circ} \mathrm{C}\end{array}$ & $<25$ jours & Dollfus 1970 \\
\hline $\begin{array}{l}\text { Dans le suc gastrique } \\
\mathrm{pH} 1 \mathrm{a} 1,5 \text { a } 37^{\circ} \mathrm{C}\end{array}$ & $>10$ jours & $\begin{array}{c}\text { Van Thiel et coll. } \\
1960\end{array}$ \\
\hline $\begin{array}{l}\text { A la chaleur } \\
60^{\circ} \mathrm{C}\end{array}$ & $<5$ minutes & Davey 1972 \\
\hline
\end{tabular}

Lorsqu'une larve d'Anisakis simplex est coupée en deux morceaux, la partie antérieure demeure encore capable de pénétrer dans la paroi du tube digestif (Asami et Inoshita, 1967). 


\section{III - Anisakidose humaine}

\section{1 - ÉPIDÉMIOLOGIE}

On relève deux caractères épidémiologiques fondamentaux :

a) Fréquence particulière dans les régions littorales de la Mer du Nord et du nord de l'Océan Atlantique européen (Pays-Bas, Écosse, sud-ouest de la Bretagne) et américain, du nord de l'Océan Pacifique en Extrême-Orient et en Amérique, ainsi qu'à Hawaï, au Chili et en Australie. Plus de 120 espèces de poissons sont parasitées par les larves d'Anisakidés. Les principales sources d'anisakidose humaine dans le monde sont reportées sur le tableau II.

Tableau II. - Principales sources d'anisakidose humaine dans le monde.

\begin{tabular}{|c|c|c|c|c|c|}
\hline Pays & Auteurs & Nom commercial & Nom latin & Paraslte & Taux d'Infest. \\
\hline Japon & $\begin{array}{l}\text { T.Oshima } \\
\text { et M. Kliks } 1986 \\
\text { T. Saito et coll. } \\
1970\end{array}$ & $\begin{array}{l}\text { maquereau japonais } \\
\text { saumon chum } \\
\text { lieu jaune } \\
\text { calmar } \\
\text { morue } \\
\text { Ilétan de l'Allantique }\end{array}$ & $\begin{array}{l}\text { Pneumalophorus japonicus japonicus } \\
\text { Onchorhynchus kela } \\
\text { Theragra chalcogramma } \\
\text { Todarodes pacilicus } \\
\text { Gadus mornua macrocephalus } \\
\text { Hippoglossus sienolepls }\end{array}$ & $\begin{array}{l}\text { Anisakis } \\
\text { Anisakis } \\
\text { Anisakis } \\
\text { Anisakis } \\
\text { P.decipiens } \\
\text { et Anisakis } \\
\text { P.decipiens }\end{array}$ & $\begin{array}{c}92,50 \% \\
100 \%\end{array}$ \\
\hline Etals-Unls & $\begin{array}{l}\text { Kliks } 1983 \\
\text { T. Deardortl 1982; } \\
1986\end{array}$ & $\begin{array}{l}\text { sébaste } \\
\text { saumon aflantique } \\
\text { "snapper" }\end{array}$ & $\begin{array}{l}\text { Sebastes spp } \\
\text { Salmo salar } \\
\text { Lutjanidés }\end{array}$ & $\begin{array}{l}\text { P.decipiens } \\
\text { et Anisakis } \\
\text { P.decipiens }\end{array}$ & $59-96 \%$ \\
\hline Chiti & $\begin{array}{l}\text { J.Carvajal et } \\
\text { P.E. Cattan } 1979\end{array}$ & merlu & Merluccius gayi & $\begin{array}{l}\text { Anisakis el } \\
\text { P.decipiens }\end{array}$ & $\begin{array}{l}90,50 \% \\
10,80 \%\end{array}$ \\
\hline $\begin{array}{l}\text { Atlantique Nord } \\
\text { et Arctique }\end{array}$ & N.E.Platt 1975 & morue & Gadus morhua & $\begin{array}{l}\text { P.decipiens } \\
\text { et Anisakis }\end{array}$ & $70-95 \%$ \\
\hline Yougoslavie & J.Brglez 1985 & $\begin{array}{l}\text { maquereau } \\
\text { morue }\end{array}$ & $\begin{array}{l}\text { Scomber scomber } \\
\text { Gadus spp. }\end{array}$ & $\begin{array}{l}\text { Anisakis } \\
\text { Anisakis }\end{array}$ & $80 \%$ \\
\hline Italie & $\begin{array}{l}\text { A. Panebianco et coll. } \\
1985\end{array}$ & $\begin{array}{l}\text { hareng fumé imponé } \\
\text { des Pays-Bas }\end{array}$ & Clupea harengus & Anisakis & $85,70 \%$ \\
\hline
\end{tabular}

b) Atteinte élective des consommateurs amateurs de poisson cru ou peu cuit. Le premier cas de granulome éosinophilique dû à Anisakis remonte à 1955 aux Pays-Bas. De 1955 à 1968, 160 autres cas, probables ou confirmés ont été rapportés aux Pays-Bas, dus à la consommation de hareng "vert "(= fumé). Ce chiffre a diminué depuis l'apparition d'une réglementation sanitaire (Van Thiel, 1976). 
$\mathrm{Au}$ Japon, où les plats de poissons crus sont traditionnels (sashimi, sushi, ceviche, oka, etc.), l'anisakidose est extrêmement répandue ; Ishikura et coll. recensent 1523 cas d'anisakidose gastrique et 223 anisakidoses intestinales en 1983 (Oshima, 1987).

Aux États-Unis, 25 cas ont été signalés ; Smith et Wootten (1978) estiment qu'ils sont dus au goût pour les plats étrangers et au " mouvement vers une alimentation naturelle ".

A Taïwan (Chine), les plats de poissons crus sont aussi traditionnels, et il existe 13 espèces de poissons fortement parasités par Anisakis sp. (37\% de poissons parasités, avec un taux moyen de 14,2 larves par poisson, ce taux atteignant 80,3 larves par poisson dans certaines espèces). Cependant, l'anisakidose n'est pas fréquente, parce que les Chinois mangent le poisson cru à la fin du repas, et risquent donc moins de s'infester que les Japonais qui le mangent au début du repas quand l'estomac est vide (Chao, 1985).

\section{2 - PATHOGÉNIE}

La plupart des larves trouvées chez l'homme sont des Anisakis simplex et des Pseudoterranova decipiens (Oshima, 1987). Existent aussi de rares cas dont les larves ne sont pas identifiables; on suspecte le genre Contracaecum (Mudry et coll., 1986). Un seul cas d'anisakidose confirmée dû à la iarve de Contracaecum osculatum a été rapporté par Schaum et Muller (cité par Weerasoorlya, 1986). Les larves d'Hysterothylactum n'ont jamais été retrouvées, et leur cas sera discuté dans la deuxième partie.

Quand la L3 d'Anisakis simplex est ingérée vivante par l'homme, elle cherche toujours à pénétrer dans la paroi du tube digestif. Cela provoque une lésion traumatique et cause une violente douleur accompagnée d'autres symptômes (hémorragies, inflammation, ulcérations, etc.). Puis une réaction inflammatoire non spécifique entraîne la mort de la larve.

S'il s'agit d'une réinfestation, une réaction antigène-anticorps révèle l'endroit de la pénétration de la larve et entraîne la formation d'un granulome éosinophilique ou phlegmonneux allergique (type Arthus), ce qui s'observe souvent dans les cas chroniques.

L'hypersensibilité peut être due aux substances excrétées-sécrétées par les larves vivantes, mais aussi aux produits de la décomposition des larves mortes dans les tissus (Smith et Wootten, 1978).

\section{3 - SYMPTOMES ET LESIONS}

\section{a) Signes Cliniques}

On distingue deux types d'anisakidoses, qui se manifestent par des symptòmes différents. 
1) Anisakidose gastrique

Forme aiguë en général. Un syndrome abdominal peut survenir brutalement, quelques heures seulement (1 à 7 heures) après l'ingestion du poisson parasité ; douleurs violentes, nausées et vomissements. Un examen gastroscopique peut permettre d'observer la larve vivante en train de pénétrer dans la paroi de l'estomac.

Mais la plupart des cas sont difficiles à diagnostiquer à cause de l'absence ou de la discrétion des symptômes. Certains cas peuvent être confondus avec des ulcères, cancers, ou polypes de l'estomac. En un an, dans un hôpital au Japon, Oshima (1972) a relevé 153 cas de diagnostics erronés d'anisakidose gastrique.

En l'absence de diagnostic rapide, la maladie devient chronique; alors une douleur sourde peut persister de quelques mois à une ou plusieurs années. Le taux sanguin d'éosinophiles passe de 4 à $40 \%$ dans plus de la moitié des observations, mais la leucocytose reste très faible ou absente (Oshima, 1972). Dans $70 \%$ des cas, Yokogama et Yoshimura (1967) signalent la présence de sang dans le suc gastrique. Chez beaucoup de patients apparaît une diminution, ou même une disparition, de l'acidité gastrique. 11 est possible que des modifications quantitatives de la sécrétion gastrique favorisent la pénétration des larves dans la paroi de l'estomac (Asami et Inoshita, 1967).

L'anisakidose gastrique est souvent provoquée par les larves de Pseudolerranova decipiens, comme cela est observé au Japon (Oshima, 1987). Les anisakidoses dues aux L3 de $P$. decipiens se manifestent par une douleur épigastrique intermittente toutes les 5 minutes, avec nausées et vomissements. Quelquefois, apparaissent de l'urticaire et des picotements à la gorge.

Dans de rares cas au Japon, on trouve la larve par endoscopie sans aucun symptôme (Oshima, 1987). Aux États-Unis, on rapporte 3 cas sans symptôme abdominal, les larves étant mises en évidence dans la bouche ou la gorge. Les vers trouvés chez l'homme sont non seulement des L3, mais aussi des IA et même des adultes (Little et coll., 1973 ; Kates et coll., 1973 ; Lichtenfels et coll., 1976 ; Kliks, 1983).

Dans un cas dù à des L3 d'Anisakis sp., a évolué une polyarthrite aiguë, en relation avec l'apparition de complexes immuns (Fabresse et coll., 1984).

2) Anisakidose intestinale

30 à $40 \%$ des cas d'anisakidose concernent l'intestin, souvent au niveau de l'iléon (Oshima et Kliks, 1986). Le symptôme typique est la douleur abdominale aiguë, qui apparaît tout à coup, 1 à 5 jours après l'ingestion de poisson cru, avec nausées, vomissements et diarrhée. L'éosinophilie sanguine reste souvent normale, par contre la leucocytose est marquée dans de nombreux cas. L'irritation du péritoine et de l'intestin grêle est souvent signalée. Les lésions peuvent être mises en évidence lors d'une laparotomie, avec présence possible de 200 à $500 \mathrm{ml}$ de liquide jaunâtre dans la cavité péritonéale (Oshima, 1972, 1987).

A l'examen histologique, la larve entière ou en morceaux peut être observée au centre de la lésion. Le phlegmon éosinophilique typique est retrouvé avec un œdème de la paroi intestinale (Van Thiel, 1975). 
L'anisakidose intestinale est due souvent à la L3 d'A. simplex et très rarement à $P$. decipiens (un cas est rapporté par Seo, 1984, cité par Oshima, 1987).

3) Autres localisations

Smith et Wootten (1978) ont signalé que dans certains cas, les larves peuvent pénétrer dans un ganglion lymphatique, le pancréas, l'épiploon et le mésentère, et même dans la paroi du pharynx.

\section{b) Caractères histologiques}

L'étude histologique de la pièce opératoire montre en général une infiltration massive par des polynucléaires éosinophiles, dans la muqueuse, la sous-muqueuse, ou la séreuse. Au centre du granulome on retrouve parfois le parasite ou ses débris, ou un foyer de nécrose. Quelquefois, il existe des ulcérations muqueuses profondes, comblées par un bourgeon charnu contenant des cellules géantes. D'après les chercheurs japonais, il y a 5 types de lésions dans l'anisakidose (Oshima, 1972).

1) Réaction de type corps étranger : fréquente dans l'anisakidose gastrique.

2) La réaction "phlegmoneuse" (type Arthus) peut être fréquemment observée dans les cas d'anisakidose intestinale.

3) Lésion de type abcès possible dans les cas chroniques.

4) "Abcès-granulome " lors d'évolution de durée supérieure à 6 mois.

5) Granulome dans les lésions les plus anciennes ; ce type correspond au remplacement total de l'abcès par un tissu granulomateux, avec infiltration éosinophilique. On ne retrouve que des débris larvaires.

\section{4 - DIAGNOSTIC}

\section{a) Diagnostic clinigue}

Une enquête sur l'alimentation du patient permet de préciser s'il est un consommateur habituel de poisson cru ou peu cuit, et s'il a ressenti une douleur épigastrique après un repas de poisson.

Dans le cas de douleur épigastrique, le diagnostic peut être confirmé par mise en évidence du parasite en se servant d'un fibroscope. On observe souvent une larve blanc jaunâtre (Anisakis) ou rougeâtre ( $P$. decipiens) plantée dans une zone œdématiée, avec un abondant exsudat et même du sang. Le traitement peut être aussitôt entrepris par l'enlèvement des larves avec une pince à biopsie. Actuellement, l'utilisation de l'endoscopie en clinique montre, notamment au Japon, une grande efficacité dans le diagnostic et le traitement de l'anisakidose (Fujino et coll., 1984).

Par contre, des difficultés subsistent pour l'anisakidose intestinale, dont les symptômes prêtent souvent à confusion avec divers syndromes abdominaux (entérite, appendicite, occlusion intestinale, etc.). Le diagnostic n'est souvent confirmé qu'après un examen histologique pratiqué à la suite d'une laparotomie. 
L'examen radiographique peut quelquefois mettre en évidence la larve en utilisant une technique de visualisation de la muqueuse par produit de contraste (" mucal relief method", Oshima, 1972).

\section{b) Diagnostic immunologique}

Diverses méthodes d'immunodiagnostic ont donné de bons résultats :

- Hémagglutination indirecte (Suzuki et coll., 1975).

— Intra-dermo réaction (Kobayashi et coll., 1968).

- Immuno-fluorescence indirecte (Suzuki et coll., 1974).

- Ouchterlony, électrosynérèse et immunoélectrophorèse (Petithory et coll., 1986).

- R. A. S. T. (test de " radioallergosorbant ») (Desowitz, 1985).

Les antigènes $(\mathrm{Ag})$ utilisables sont variés : $\mathrm{Ag}$ somatiques ou " $\mathrm{Ag}$ som. " (extrait total de L3) ; Ag excrétés-sécrétés pendant la mue des L3, ou " Ag E. S. "; hémoglobine de L3 couplée à un élément stabilisateur.

Kobayashi et coll. (1968) ont signalé que, dans les examens par intradermoréaction effectués sur 769 personnes, 10,5\% sont positives avec $\mathrm{Ag}$ E. S., et $4,7 \%$ avec Ag.som.

L'antigène hémoglobine cỏuplée est stable et sensible pour l'immuno-fluorescence indirecte. Les réactions croisées avec les autres helminthoses sont faibles et l'examen est très spécifique (Suzuki et coll., 1974).

L'immuno-diagnostic de l'anisakidose est utilisable au cours d'enquêtes épidémiologiques (Kobayashi et coll., 1968), mais pas encore pour le diagnostic clinique quotidien, hormis les cas d'anisakidose chronique (Godeau et coll., 1985 ; Petithory et coll., 1986). En effet les anticorps apparaissent très tardivement après l'infestation.

c) Diagnostic post-opératoire

11 permet de confirmer la présence de larves ou de fragments de larves dans les tissus.

\section{5 - TRAITEMENT ET PROPHYLAXIE}

Le traitement est effectué en même temps que le diagnostic par extraction de la larve dans les cas d'anisakidose aiguë gastrique ou duodénale. Les symptômes disparaissent alors rapidement en quelques jours (Oshima, 1987).

Dans les cas chroniques, l'efficacité des thérapeutiques médicales utilisant un anthelminthique et un médicament anti-inflammatoire reste à déterminer (Mudry et coll., 1985). L'exérèse de la lésion contenant les larves est nécessaire pour prévenir une réaction aux antigènes larvaires et une exacerbation allergique future des lésions (Van Thiel, 1975).

Depuis 1968, une diminution des cas d'anisakidose a été obtenue aux Pays- 
Bas grâce à une législation prévoyant la congélation obligatoire des poissons à $-20^{\circ} \mathrm{C}$ pendant 24 heures, avant leur préparation pour le salage ou le fumage. Au Japon, cette mesure est impraticable, car rejetée par les consommateurs, et le nombre de cas augmente toujours : 1000 à 2000 cas environ suspectés chaque année, mais Oshima (1987) considère que l'augmentation apparente du nombre de cas au Japon résulte plutôt d'une amélioration du diagnostic grâce à l'endoscopie, que d'infestations plus fréquentes. Dans les autres pays, la seule mesure prophylactique est de proposer la congélation des poissons au moins 24 heures à $-20^{\circ} \mathrm{C}$, délai porté à 5 jours pour les sébastes (Oshima, 1987 ; Deardoff et coll., 1984).

L'importance et la gravité de ces problèmes chez l'homme, ainsi que la rareté des informations sur la situation en France, justifiaient une enquête sur les larves présentes chez les poissons couramment consommés. Nous avons entrepris cette enquête dans la région parisienne en 1986 et 1987 ; les résultats en seront présentés dans la deuxième partie. 